\title{
Seguimiento de la frecuencia cardiaca y la presión parcial de oxígeno durante la cirugía bucal
}

\author{
Arias Chamorro $B^{*}$, Romero Olid $M N^{* *}$, Hita Iglesias $C^{*}$, Bravo Pérez $M^{* * *}$, \\ Vallecillo Capilla $M * * * *$
}

\begin{abstract}
RESUMEN
Se hicieron mediciones de la frecuencia cardiaca y la presión parcial de oxígeno en sangre a un grupo de 29 pacientes durante la cirugía bucal. El objetivo del estudio era comprobar si había diferencias estadísticas entre los datos obtenidos en cada momento quirúrgico. Aplicando el test de student para muestras apareadas se obtuvieron los siguientes resultados:

1. - La frecuencia cardiaca se elevó de manera significativa tras la anestesia y se mantuvo durante el despegamiento. Volvió a descender durante la osteotomía, manteniéndose durante la sutura y en el alta. En estos momentos no había diferencias con el momento inicial.

2. - La presión parcial de oxígeno en sangre permaneció casi constante en cada tiempo de la cirugía bucal, y no hubo variaciones que pudieran ser atribuibles a un momento específico de la misma.
\end{abstract}

Palabras clave: cirugía oral; frecuencia cardiaca; presión parcial de oxígeno.

\section{SUMMARY}

Measures of heart rate and oxygen partial pressure were made in a group of 29 patients during oral surgery. The aim of the study was to compare data coming from every surgical moment by statistical methods. By using student test for coupled samples the next results were found:

1. - Heart rate increased significantly after anesthesia and went on while separating of the flap. After that, it decreased during osteotomy and didn't show considerable changes during the closing of the wound and the discharging of the patient. At this point, there were not differences compared with the starting moment.

2. - Oxygen partial pressure in blood remained almost constant while the oral surgery and there were not variations that could be linked to an specific stage of the ongoing surgery.

Key words: oral surgery; heart rate; oxygen partial pressure.

* Alumno de Master de Cirugía Bucal e Implantología de la Facultad de Odontología de la Universidad de Granada.

** Profesora Asociada de Cirugía Bucal y Maxilofacial de la Facultad de Odontología de la Universidad de Granada.

*** Profesor Titular de Odontología Preventiva y Comunitaria de la Facultad de Odontología de la Universidad de Granada.

**** Profesor Titular de Cirugía Bucal y Maxilofacial de la Facultad de Odontología de la Universidad de Granada.

Arias Chamorro B, Romero Olid MN, Hita Iglesias C, Bravo Pérez M, Vallecillo Capilla M. Seguimiento de la frecuencia cardiaca y la presión parcial de oxígeno durante la cirugía bucal . Av. Odontoestomatol 2003; 19-2: 7580. 


\section{INTRODUCCIÓN}

La frecuencia cardiaca es uno de los parámetros a considerar en la exploración clínica de un paciente. La aparición de una taquicardia o una bradicardia extrema podría dar lugar a consecuencias fatales ${ }^{[1,2,3]}$. Por esto, hay que tener muy presentes los factores que puedan inducir un incremento o un descenso en el ritmo cardiaco, así como las enfermedades sistémicas que puedan hacer al paciente más sensible a estos cambios ${ }^{[4,5,6,7]}$.

Sabemos que el estrés psicológico, un estímulo doloroso o la presencia de simpaticomiméticos en el anestésico local pueden incrementar la presión arterial y la frecuencia cardiaca ${ }^{[8,9,10,11,12,13]}$. Por tanto, los mecanismos responsables de su elevación durante la cirugía oral parecen implicar total o parcialmente al sistema nervioso autónomo, aunque sus cambios específicos no se conocen con exactitud ${ }^{[14,15]}$.

La presión parcial de oxígeno $\left(\mathrm{PpO}_{2}\right)$ en la gasometría es un parámetro bioquímico clave a seguir en pacientes sometidos a intervenciones quirúrgicas. Se mide en sangre arterial, y la disminución de su valor de forma aguda puede darnos la señal de la aparición de una urgencia que comprometa la vida del paciente. Su valor normal es de $95 \mathrm{~mm}$ de $\mathrm{Hg}$ aunque puede mantenerse sin que suponga patología entre 85 y $100 \mathrm{~mm}$ de $\mathrm{Hg}$, incluso en enfermos crónicos hasta $60 \mathrm{~mm}$ de $\mathrm{Hg}$. La $\mathrm{PpO}_{2}$, influenciada por la de anhídrido carbónico y por el $\mathrm{pH}$, refleja la saturación de la hemoglobina y por tanto el aporte de oxígeno a los tejidos.

La $\mathrm{PpO}_{2}$ disminuye (hipoxemia) por múltiples causas que pueden resumirse en el trastorno de los tres grandes mecanismos de la respiración: la ventilación, la perfusión sanguínea alveolar y la difusión de gases entre capilares y alveolos.

- Trastornos de la ventilación: sus causas pueden ser de origen neurológico por uso de medicamentos como hipnóticos, anestésicos o analgésicos; o por accidentes cerebrovasculares que afecten al centro respiratorio y por tanto a la movilidad torácica. También puede originarse una hipoxemia aguda de origen broncopulmonar por estenosis u oclusión de las vías respiratorias, ya sea por reacciones alérgicas (anafilaxias y edemas de glotis) o por cuerpos extraños. Otras causas de hipoxemia de origen ventilatorio serían el uso de fármacos curarizantes que podrían ocasionar una parálisis súbita de los músculos respiratorios y la aparición de un neumotórax espontáneo que disminuye la capacidad vital pulmonar.

- Trastornos de la perfusión sanguínea alveolar: en infartos cardiacos y en embolias pulmonares.

- Trastornos de la difusión a través de la membrana alveolocapilar: en edema agudo de pulmón por insuficiencia cardiaca, en intoxicaciones y en las alergias agudas.

El objetivo de este estudio fue valorar la influencia de cada momento de una intervención quirúrgica bucal sobre la frecuencia cardiaca (FC) y la $\mathrm{PpO}_{2}$ en sangre, y observar si pudiese existir una influencia específica de cada fase de la misma sobre el sistema vegetativo y su repercusión en el pulso o sobre la saturación de oxígeno. Pretendíamos comprobar si había diferencias estadísticamente significativas entre los datos obtenidos en cada momento de la cirugía, que pudiesen indicar una acción mediadora del sistema nervioso autónomo o el endocrino, en momentos distintos a los que cabría esperar debidos a la anestesia o al estrés.

\section{MATERIAL Y MÉTODOS}

Hemos evaluado en este estudio una muestra de 29

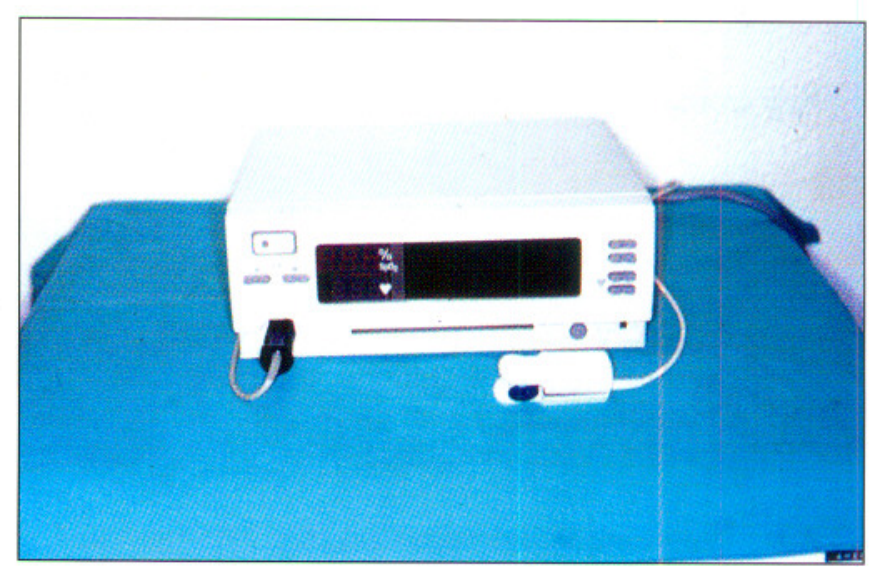

Fig. 1. Ohmeda-3800 pulse oximeter: pulsiosímetro utilizado para la medición de la frecuencia cardiaca y la presión parcial de oxígeno en sangre. 
Arias Chamorro B, Romero Olid MN, Hita Iglesias C, Bravo Pérez M, Vallecillo Capilla M Seguimiento de la frecuencia cardiaca y la presión parcial de oxígeno durante la cirugía bucal

pacientes intervenidos en el Máster de Cirugía Bucal de la Facultad de Odontología de la Universidad de Granada. El rango de edad de los mismos osciló entre los 12 y 54 años, siendo la media de 27.3, y del total de los pacientes, el $62.1 \%$ fueron hembras.

A cada uno de los pacientes se le realizó una Historia Clínica en la cual se incluía una parte general en la que se refleja la filiación, antecedentes personales y familiares y otra parte más específica que incluye una anamnesis de la cavidad oral. Se realizó una exploración clínica bucal y se solicitó una ortopantomografía para completar el diagnóstico de la región a intervenir.

Los datos de la historia clínica más significativos podrían resumirse en los siguientes:

- El $41 \%$ de los pacientes evaluados eran fumadores, incluyendo los esporádicos (se denominó fumador al que consumía más de 4 cigarrillos / día).

- El 93.1\% no presentaban ninguna cardiopatía previa, y sólo se registró en un paciente un soplo funcional e hipotensión en otro.

- El 79.3\% no tomaban medicación previa, y en el resto, era muy variada.

- Para el $34.5 \%$ ésta era su primera cirugía, y del resto el $37.9 \%$ ya había pasado una cirugía oral y el $27.6 \%$ en otra región corporal.

- El 72.4\% mantenían una vida sedentaria.

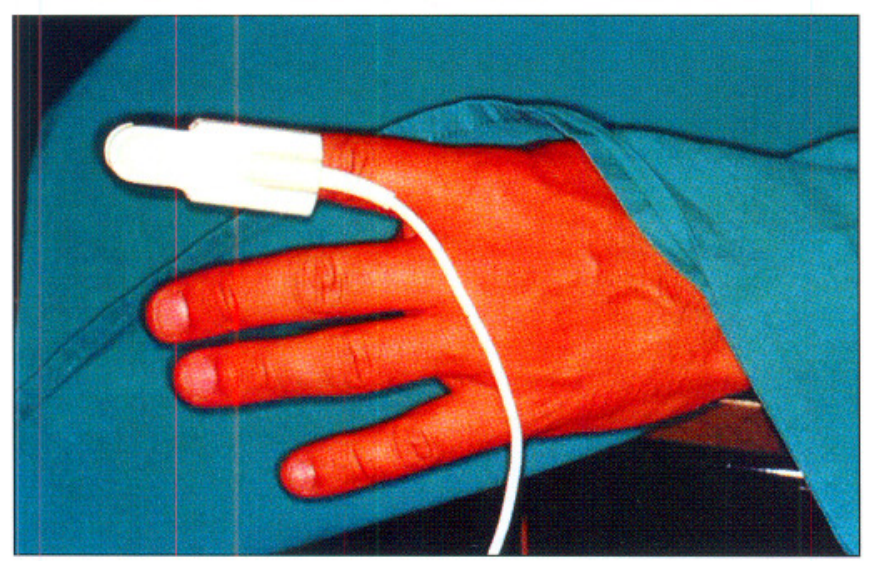

Fig. 2. Sensor digital tipo pinza que conecta el dedo índice de la mano izquierda con el pulse oximeter.
Todos los pacientes estuvieron en decúbito desde la primera toma de datos unos 3 minutos antes de la cirugía, con los ojos y el cuerpo tapados con paños estériles. Siempre hubo al menos 2 operadores durante el acto quirúrgico, si bien los registros los realizó siempre la misma persona.

Ningún paciente recibió más de 2 carpules de anestesia (ULTRACAIN" ${ }^{*}$ - 40,0 mg de articaina y 0,01 mg de epinefrina por ml de solución).

La aparatología que se utilizó en el estudio fue el pulsiosímetro OHMEDA- 3800 PULSE OXIMETER (Fig.1), que está instalado en el quirófano y que tiene la capacidad de medir la frecuencia cardiaca y la presión parcial de oxigeno en sangre periférica en cada momento. El aparato puede programarse para que suene la alarma cuando la frecuencia o la saturación de $\mathrm{O}_{2}$ pase unos límites establecidos. El sensor tiene un diseño tipo pinza que se fija en el dedo índice de la mano izquierda de cada paciente (Fig. 2).

La toma de datos se hizo de la siguiente manera:

- Toma inicial o basal: se midió 3 veces la frecuencia cardiaca y la presión de oxígeno unos 3 minutos antes de la intervención, estando ya el paciente tumbado, relajado y tapado.

- Toma tras la anestesia: se tomaron otros 3 datos una vez puesta la anestesia desde el momento en que el paciente refiere no sentir el tacto mucoso y antes de la incisión.

- Toma del despegamiento del colgajo: 3 datos durante el levantamiento de la fibromucosa con el periostotomo.

- Toma de la osteotomía: 3 datos en el momento de la osteotomía.

- Toma de la sutura: otros 3 datos mientras se suturaba.

- Toma en el alta: otros 3 datos cuando el paciente comprende que la cirugía ha terminado, ya destapado, con ojos descubiertos y antes de ser incorporado.

Se hizo la media a cada trío de datos provenientes de 
cada momento de la intervención, obteniéndose un único dato para cada tiempo quirúrgico. De esta manera, de 18 datos iniciales (3 tomas por 6 momentos quirúrgicos) sacamos 6 datos medios por paciente.

\section{RESULTADOS}

Se realizó la media para cada momento de la cirugía de los datos medios obtenidos en cada paciente.

En la tabla 1 podemos observar la variable de las

\begin{tabular}{|c|c|c|}
\hline \multicolumn{3}{|c|}{$\begin{array}{l}\text { TABLA 1.- FRECUENCIAS CARDIACAS } \\
\qquad(n=29 \text { pacientes })\end{array}$} \\
\hline & $\begin{array}{c}\bar{X} \\
\text { (media) }\end{array}$ & $\begin{array}{c}\text { de } \\
\text { (desviación estándar) }\end{array}$ \\
\hline Inicial & 87,5 & $(15,8)$ \\
\hline Tras anestesia & 96,1 & $(15,2)$ \\
\hline Durante despegamiento & 93,3 & $(19,9)$ \\
\hline Durante osteotomía & 89,9 & $(17,6)$ \\
\hline Durante sutura & 86,0 & $(16,9)$ \\
\hline $\mathrm{Al}$ alta & 86,7 & $(16,4)$ \\
\hline
\end{tabular}

\begin{tabular}{|c|c|c|c|}
\hline \multicolumn{4}{|c|}{$\begin{array}{l}\text { TABLA 2.- FRECUENCIAS CARDIACAS } \\
\text { ( } n=29 \text { pacientes })\end{array}$} \\
\hline Variable & $\bar{X}$ & de & $\begin{array}{c}\text { Comparación } \\
\text { por parejas }\end{array}$ \\
\hline Inicial & 87,5 & $(15,8)$ & $\nabla$ \\
\hline Tras anestesia & 96,1 & $(15,2)$ & $\| v$ \\
\hline $\begin{array}{l}\text { Durante } \\
\text { despegamiento }\end{array}$ & 93,3 & $(19,9)$ & mav \\
\hline Durante osteotomía & 89,9 & $(17,6)$ & 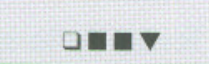 \\
\hline Durante sutura & 86,0 & $(16,9)$ & पenev \\
\hline $\mathrm{Al}$ alta & 86,7 & $(16,4)$ & בaman \\
\hline
\end{tabular}

La simbología debe leerse en vertical; así $\mathbf{\nabla}$ @ significa $p<0.05$ es decir comparación estadísticamente significativa; $\mathrm{y} \mathbf{\nabla} \sqsupset$ significa comparación no significativa estadísticamente $(p>0.05)$. Comparaciones mediante test de student para muestras apareadas. fases de la cirugía en la columna de la izquierda, la media de las frecuencias cardiacas en cada momento de la misma en la columna del centro, y las desviaciones estándar (de) de los datos tomados en la columna de la derecha. Cada valor de media de la tabla proviene de 87 registros de frecuencia cardiaca en cada momento de la cirugía ( $n=29$ pacientes por 3 tomas en cada momento).

En la tabla 2 se realizan comparaciones entre las medias de la tabla anterior, aplicando el test de student para muestras apareadas. Con éste, comparamos si la frecuencia cardiaca en cada momento de la cirugía, varía en términos estadísticos respecto a cada una de las otras, obtenidas en los otros momentos quirúrgicos.

De este modo, analizando la columna de la derecha en esta segunda tabla, en la cual se muestran los resultados de las comparaciones se podrían dar los siguientes resultados:

1- Hay diferencias entre la FC inicial y las que se obtienen tras la anestesia y el despegamiento.

2- No hay diferencias entre la FC inicial y las que provienen de la osteotomía, de la sutura y del alta. Tampoco entre las FC tras la anestesia y durante el despegamiento.

\begin{tabular}{|l|ccc|}
\hline \multicolumn{4}{|c|}{ TABLA 3.- PRESIÓN PARCIAL DE OXÍGENO } \\
( $\mathrm{n}=29$ pacientes)
\end{tabular}

La simbología debe leerse en vertical; así $\mathbf{\nabla}$. significa $p<0.05$, y $\mathbf{v} \square$ significa comparación no significativa estadísticamente $(p>0.05)$. Comparaciones mediante test de student para comparaciones apareadas. 
3- Empieza a haber diferencias entre el despegamiento y la osteotomía y se mantiene hasta la sutura.

Los resultados muestran que la frecuencia cardiaca basal se eleva tras la anestesia, se mantiene durante el despegamiento, y comienza a descender durante la osteotomía. En el momento del alta no parece haber diferencias con las tomas iniciales.

En la tabla 3 se aplican las mismas comparaciones que en la tabla anterior entre las medias de las presiones parciales de $\mathrm{O}_{2}$. Analizando el resultado de las comparaciones no hay diferencias significativas entre las medias de presiones parciales de $\mathrm{O}_{2}$ en cada momento de la cirugía.

Discusión: Diferentes autores han estudiado la frecuencia cardiaca, la presión arterial y la presión parcial de $\mathrm{O}_{2}$ en sangre durante la cirugía oral.

Matsumura y cols., estudiaron la frecuencia cardiaca y la presión arterial durante la cirugía bucal, observando que tras la administración del anestésico local ambos parámetros se elevaban; y que esta elevación era más acusada en los pacientes con más de 40 años. En los jóvenes, el sistema nervioso vegetativo parecía responder más suavemente durante el acto quirúrgico ${ }^{|14|}$.

Abraham-Inpijn y cols., midieron también la frecuencia cardiaca con un electrocardiógrafo y la presión arterial durante tratamientos dentales con anestesia local y encontraron también elevación de estos parámetros tras la infiltración ${ }^{[8]}$. Coincidiendo con estos autores Gortzak y cols., también obtuvieron resultados similares en tratamientos dentales rutinarios con anestesia local ${ }^{[9]}$.

Tsuchihashi y cols., realizaron un seguimiento en intervenciones quirúrgicas bucales de la presión arterial y de la frecuencia cardiaca, obteniendo elevaciones similares a las comentadas ${ }^{[10]}$.

Kohler-knoll y cols., encontraron un incremento de la frecuencia cardiaca y una elevación de las catecolaminas y sus metabolitos en suero en las extracciones de terceros molares inferiores bajo anestesia local ${ }^{[11}$.

Los resultados de nuestro estudio coinciden bási- camente con los hallados en la literatura. La frecuencia cardiaca se incrementa durante la cirugía oral, por el efecto taquicárdico del anestésico local y su vasoconstrictor o por la actividad vegetativa del estrés quirúrgico. Sin embargo en nuestro estudio, la taquicardia cede a niveles no significativos durante la osteotomía sin medir el tiempo de duración quirúrgica, aunque se mantiene el efecto anestésico. Por esto, cabe pensar que el paciente una ver tranquilizado tras el comienzo de la cirugía atenúa en parte el efecto elevador del anestésico o que la fibromucosa muestra una sensibilidad especial en la incisión y despegamiento en términos vegetativos.

Respecto a la variación de la presión parcial de $\mathrm{O}_{2}$ en sangre durante la cirugía bucal, nuestros resultados coinciden con otros autores consultados. La presión parcial de $\mathrm{O}_{2}$ permanece prácticamente invariable a lo largo de la cirugía. Esto es lógico siempre que no suceda una urgencia como las que se han comentado en las causas de hipoxemia. Por tanto no parece haber una relación entre la cirugía bucal y la presión parcial de $\mathrm{O}_{2}$ en sangre.

\section{Podrían darse las siguientes conclusiones:}

1. - Hay una elevación significativa de la frecuencia cardiaca en la cirugía oral tras la colocación de la anestesia, y se mantiene durante la incisión y el despegamiento de la fibromucosa. Aunque se mantiene el efecto anestésico, durante la osteotomía se observa una reducción del ritmo prácticamente a niveles iniciales, y se mantiene así durante la sutura y el alta del paciente.

2. - La presión parcial de oxígeno en sangre permanece invariable en cada momento de la cirugía oral, siempre que no haya alguna urgencia que ocasione hipoxemia.

\section{BIBLIOGRAFÍA}

1. Yoshimura Y. The relation of tooth extraction to anginal attack. J Oral Maxillofac Surg 1983; 41: 365-76. 
2. Okada Y, Suzuki H, Ishiyama I. Fatal subarachnoid haemorrhage associated with dental local anesthesia. Aust Dent J 1989; 34: 323-25.

3. Hunter PL. Cardiac arrest in the dental surgery. Br Dent J 1991; 170: 284.

4. Bandl E, Boda K, Sonkodi S. Hypertension screening in a dental surgery: a Hungarian study. J hum hypertens 1990; 4: 253-57.

5. Meiller TF, Overhoser CD, Kutcher MJ, y col. Blood pressure fluctuations in hypertensive patients during oral surgery. J Oral Maxillofac Surg 1983; 41: 715-18.

6. Meyer F-U. Hemodynamic changes of local dental anesthesia in normotensive and hypertensive subjects. Int J Clin Pharmacol Ther Toxicol 1986; 24: 477-81.

7. Vanderheyden PJ, Willians RA, Sims TN. Assessment of ST segment depression in patients with cardiac disease after local anesthesia. J Am Dent Assoc 1989; 119: 407-12.

8. Abraham-Inpijn L, Borgmeijer-Hoelen A, Gortzak RAT. Changes in blood pressure, heart rate, and electrocardiogram during dental treatment with use of local anesthesia. J Am Dent Assoc 1988; 116: 531-36.

9. Gortzak RAT, Oosting J, Abraham-Inpijn L. Blood pressure response to routine restorative dental treatment with and without local anesthesia. Oral Surg Oral Med Oral Pathol 1992; 73: 677-81.

10. Tsuchihashi T, Takata Y, Kurokawa H, y col. Blood pressure response during dental surgery. Hypertens Res 1996; 19: 189-94.
11. Kohler-Knoll E, Knoller M, Brandt K, y col. Cardiohemodynamic and serum catecholamine response to surgical removal of impacted mandibular third molars under local anesthesia: a randomized double-blind parallel group and crossover study. J Oral Maxillofac Surg 1991;49:95762.

12. Davenport RE, Porcelli RJ, Iacono VJ, y col. Effects of anesthetics containing epinephrine on catecholamine levels during periodontal surgery. J Periodontol 1990; 61: 553-58.

13. Salonen M, Forssell H, Scheinin M. Local dental anesthesia with lidocaine and adrenaline: effects on plasma catecholamines, heart rate and blood pressure. J Oral Maxillofac Surg 1988; 17: 39294.

14. Matsumura-K, Takata-Y, Kurokawa-H, Kajiyama$M$, Abe-I, Fujishima-M. Changes in blood pressure and heart rate variability during dental surgery. Am J Hypertens. 1998 ; 11 (Pt 1): 1376-80.

15. Kamath MV, Fallen EL. Power spectral analysis of heart rate variability: a noninvasive signature of cardiac autonomic function. Crit Rev Biomed Eng 1993; 21: 245-311.

\section{CORRESPONDENCIA}

Bartolomé Jesús Arias Chamorro

C/ Recogidas $n^{\circ} 49,1^{\circ}$ derecha

Teléfono: 958-252044

18002 Granada 